\title{
Liquiritin Alleviates Depression-Like Behavior in CUMS Mice by Inhibiting Oxidative Stress and NLRP3 Inflammasome in Hippocampus
}

\author{
Chang Liu $\mathbb{D}^{1},{ }^{1}$ Dai Yuan, ${ }^{1}$ Chi Zhang, ${ }^{2}$ Ye Tao, ${ }^{3}$ Ying Meng, ${ }^{1}$ Mengli Jin, ${ }^{1}$ Wu Song $\mathbb{D}^{1},{ }^{1}$ \\ Bingmei Wang $\mathbb{D}^{1}{ }^{1}$ and Lin Wei $\mathbb{D}^{1}$ \\ ${ }^{1}$ Clinical Medical College of Changchun University of Chinese Medicine, Changchun 130117, China \\ ${ }^{2}$ School of Basic Medicine, Changchun University of Chinese Medicine, Changchun 130117, China \\ ${ }^{3}$ Affiliated Hospital of Changchun University of Chinese Medicine, Changchun 130021, China
}

Correspondence should be addressed to Wu Song; 14603146@qq.com, Bingmei Wang; 345712323@qq.com, and Lin Wei; lynnw2013@sina.com

Received 13 August 2021; Revised 5 December 2021; Accepted 15 December 2021; Published 11 January 2022

Academic Editor: Weicheng $\mathrm{Hu}$

Copyright (C) 2022 Chang Liu et al. This is an open access article distributed under the Creative Commons Attribution License, which permits unrestricted use, distribution, and reproduction in any medium, provided the original work is properly cited.

Objective. Central inflammation is generally accepted to be involved in the pathology of depression. We investigated whether liquiritin exerts antidepressant effects by inhibiting central NLRP3 inflammasomes. Results. The behavioral despair model and chronic unpredictable mild stress (CUMS) model in mice were established to evaluate the antidepressant action of liquiritin. In the despair model study, liquiritin $(40 \mathrm{mg} / \mathrm{kg})$ administration reduced immobility time in tail suspension test (TST) and forced swimming test (FST) without affecting locomotion activity. In CUMS model study, liquiritin ( $40 \mathrm{mg} / \mathrm{kg}$, once daily for 4 weeks) significantly increased sucrose consumption and body weight of CUMS mice. The behavioral experiment results showed that liquiritin reduced the immobile time of CUMS mice in TST and FST, respectively, and increased the time spent and open arm entries in the elevated plus-maze (EPM) test. Further, the hippocampal superoxide dismutase (SOD) activity was increased in liquiritin-treated group, while malonaldehyde (MDA) decreased. Additionally, the hippocampal cytokines interleukin-18 (IL-18) and interleukin-1 beta (IL-1 $\beta$ ) contents were reduced in the liquiritin-treated group. Further, liquiritin downregulated the expression of NLRP3 in the hippocampus of CUMS mice rather than TLR4. Besides, NLRP3 inflammasome-associated proteins caspase-1 and ASC were also downregulated. However, liquiritin did not alter the thermal stability of NLRP3 in the cellular thermal shift assay (CETSA), suggesting that its inhibition of NLPR3 was not by direct targeting of NLRP3 protein. Conclusions. Liquiritin attenuates depression-like behavior of CUMS mice and inhibited cytokines levels triggered by NLRP3 inflammasome, suggesting the antidepressant action is, at least partially, associated with antioxidant stress and inhibition of NLRP3 inflammasome activation.

\section{Introduction}

Accumulating evidence suggests that mitigation of neuroinflammation could be a potential therapeutic approach for depression [1]. Clinical studies have shown that inflammatory cytokines in the central nervous system are significantly elevated, such as IL- $1 \beta$ and IL-6, in patients with depression. Recent studies consider that inhibition of NLRP3 inflammasome activation may be a promising strategy for the therapy of depression [2]. Liquiritin, a flavonoid isolated from the root of liquorice, has exhibited various biological activities, such as anti-inflammatory, antiaging, and antitumor activity [3-5]. The neuroprotective activity of liquiritin has attracted considerable attention. Studies have shown that liquiritin could resist the death of cortical neurons induced by glutamate and promote the axon growth of PC12 cells triggered by nerve growth factor $[6,7]$. Notably, an early study reported that liquiritin showed antidepressant effect via enhancement of 5-hydroxytryptamine and norepinephrine levels in the hippocampus, hypothalamus, and cortex [8]. In 
another study, liquiritin also showed antidepressant effects, which might be related to the defense of liquiritin against oxidative stress [9]. Although some evidence confirms the antidepressant effect of liquiritin, it is unclear whether the alleviation of central inflammation is involved in this effect. Therefore, we established an CUMS model to confirm the antidepression effects of liquiritin and explore the effects on cytokine release and NLRP3 inflammasome associate protein expression in the hippocampus.

\section{Materials and Methods}

2.1. Drugs and Reagents. Liquiritin (CAS: 551-15-5, molecular formula is shown in Figure 1(a)) and fluoxetine hydrochloride (CAS: 56296-78-7), purity $\geq 98 \%$, were purchased from Selleck China (Shanghai, China). PC12 cell was purchased from Procell Life Science\&Technology Co., Ltd. DMSO (D4540) was got from Sigma-Aldrich (Shanghai) Trading Co., Ltd. Superoxide dismutase (SOD) detection kit (A001-1-2), glutathione peroxidase (GSH-Px) detection kit (A005-1-2), and malondialdehyde (MDA) detection kit (A003-1-2) were all purchased from Nanjing Jiancheng Institute of Biological Engineering (Nanjing, China). BCA protein concentration detection kit (P0012S) and RIPA Lysis Buffer (P0013B) were purchased from Beyotime Biotechnology Co., Ltd. (Shanghai, China). Interleukin-1 beta (IL-1 $\beta$ ) detection kit (SEKM-0002), interleukin18 (IL-18) detection kit (SEKM-0019), NLRP3, and TLR4 antibody (K004108P, K003881P) were all purchased from Beijing Solarbio Technology Co., Ltd. (Beijing, China). Caspase-1, pro-caspase-1, IL-1 $\beta$, NEK7, and ASC (ab138483, ab238972, ab283818, ab133514, ab175449) antibodies were obtained from Abcam (Shanghai) Trading Co., Ltd. (Shanghai, China). $\beta$-Actin antibody (4970) and goat anti-rabbit IgG (HRP polymer) (98164) were purchased from Cell Signaling Technology (Danvers, USA).

2.2. Animals. One hundred and twenty male Kunming (KM) mice (18-22 g) were purchased from Yisi Laboratory Animals Company (Changchun, China), whose license number was SCXK (Ji)-2020-0002. Mice were housed at an ambient temperature of $21 \pm 2^{\circ} \mathrm{C}$ and a relative humidity of $45 \pm 5 \%$ with $12 \mathrm{~h}$ light-dark cycle for each day and free access to a standard chow diet and drinking water throughout the experiments.

2.3. Experimental Design. Forty mice were used to observe the effect of liquiritin on despair behavior. Mice were randomly assigned to 4 groups (10 for each group), including the control group, liquiritin group $(20 \mathrm{mg} / \mathrm{kg}$ or $40 \mathrm{mg} / \mathrm{kg})$, and fluoxetine group (positive control, $10 \mathrm{mg} / \mathrm{kg}$ ). Liquiritin or fluoxetine was injected by intragastric gavage once daily. TST, FST, and locomotor activity tests were performed on days 12, 13, and 14. The process is shown in Figure 1(b). Eighty mice were used to investigate the effect of liquiritin on depressive behavior induced by CUMS. Mice were divided into five groups with sixteen mice per group, including the control group, CUMS group, liquiritin group $(20 \mathrm{mg} / \mathrm{kg}$ or $40 \mathrm{mg} / \mathrm{kg})$, and fluoxetine group $(10 \mathrm{mg} / \mathrm{kg})$. The establishment method of CUMS model was based on the previous research [10] and was modified appropriately. Briefly, stimulus includes fasting ( $24 \mathrm{~h})$, no water ( $24 \mathrm{~h})$, clip tail ( $2 \mathrm{~cm}$ from the tip of the tail, $1 \mathrm{~min}$ ), continuous light for $24 \mathrm{~h}$, cold water swimming $\left(4^{\circ} \mathrm{C}, 5 \mathrm{~min}\right)$, hot water swimming $\left(40^{\circ} \mathrm{C}, 5 \mathrm{~min}\right)$, wet cage $(200 \mathrm{ml}$ water per cage, $24 \mathrm{~h})$, tilting cage $\left(45^{\circ}, 24 \mathrm{~h}\right)$, behavior restriction $(1 \mathrm{~h})$, and noise (30 $\mathrm{min})$. Mice were subjected to random stress twice a day for 42 days. After 14 days of CUMS, mice were given saline, liquiritin, or fluoxetine once daily for 28 days. Behavioral experiment was conducted on Day 40 to Day 42. At the end of the experiment, mice were euthanized with sodium pentobarbital $(150 \mathrm{mg} / \mathrm{kg})$, and then the hippocampal tissue was isolated quickly and homogenized in an ice bath. Ten samples from each group were used for Western blot and enzyme-linked immunosorbent assay (ELISA); the other six samples were used for immunohistochemistry (IHC). The experimental scheme is shown in Figure 2(a).

2.4. Tail Suspension Test (TST). TST refers to the literature method [11]. Briefly, $1 \mathrm{~h}$ after administration, mice were fixed with adhesive tape about $1 \mathrm{~cm}$ from the tail tip, hanging $15 \mathrm{~cm}$ away from the table. After $2 \mathrm{~min}$ of adaptation, $4 \mathrm{~min}$ of total immobility time was recorded. The standard of immobility was stopping struggling and hanging vertically.

2.5. Forced Swimming Test (FST). FST has been modified according to the documentary method [12]. In short, $1 \mathrm{~h}$ after administration, mice were placed in a Perspex bucket (height $25 \mathrm{~cm}$, diameter $12 \mathrm{~cm}$ ), filled with water to a height of $10 \mathrm{~cm}$ (water temperature was kept at $23 \pm 2^{\circ} \mathrm{C}$ ). After 2 min of adaptation, 4 min of total immobility time was recorded. The standard of immobility was stopping struggling, floating upright, or making small movements to keep the head above water.

2.6. Locomotor Activity Test. After $1 \mathrm{~h}$ of administration, mice were placed in the autonomic activity tester (Chengdu Taimeng Software Co., Ltd., China). After 2 min of adaptation, the locomotor activities of the mice were recorded by camera for $10 \mathrm{~min}$.

2.7. Sucrose Preference Test. The test was performed $1 \mathrm{~h}$ after administration. During the time, animals were given one bottle of $1 \%$ sucrose solution $(100 \mathrm{ml})$ and one bottle of distilled water $(100 \mathrm{ml})$. After $2 \mathrm{~h}$, the consumption of sucrose water and distilled water was measured. We switched the position of the bottles at the midpoint of the test to avoid possible side effects. Sucrose preference is measured as the percentage of sucrose solution consumed relative to total liquid intake. The test was conducted every 7 days.

2.8. Elevated Plus-Maze (EPM). The elevated plus-maze consists of two open arms and two closed arms (arm length $30 \mathrm{~cm}$, arm width $6 \mathrm{~cm}$, elevated height $50 \mathrm{~cm}$ ) with a central area of $5 \times 5 \mathrm{~cm}$. At the beginning of the test, mice were placed on a central platform with their heads towards one open arm. 


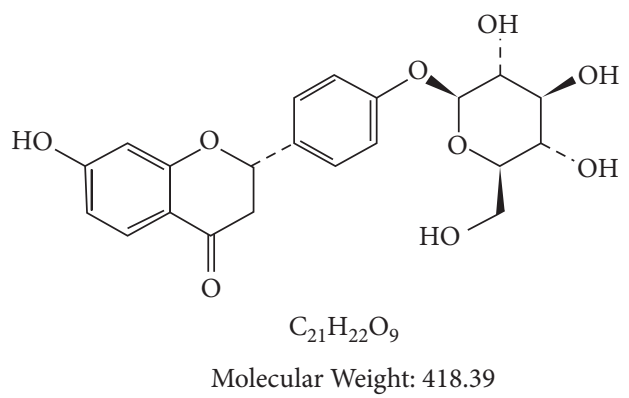

(a)

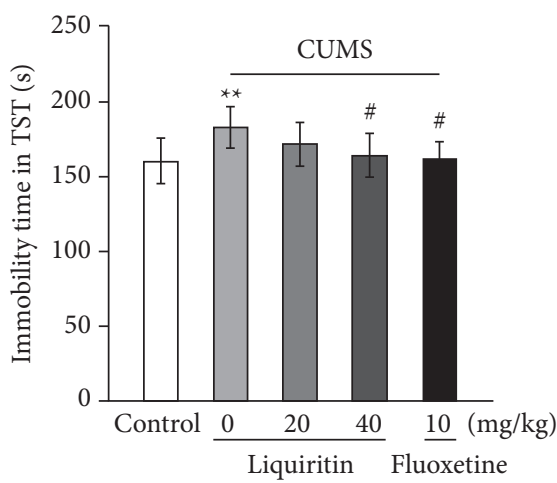

(c)

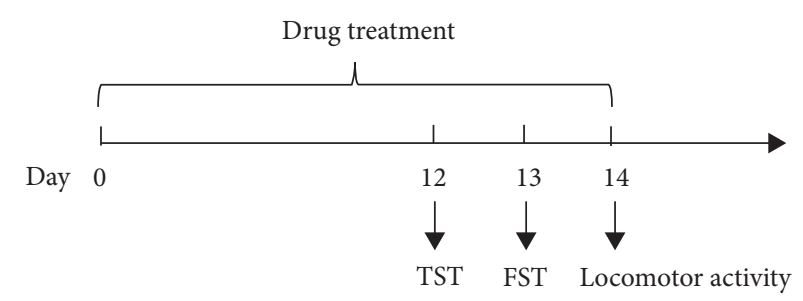

(b)

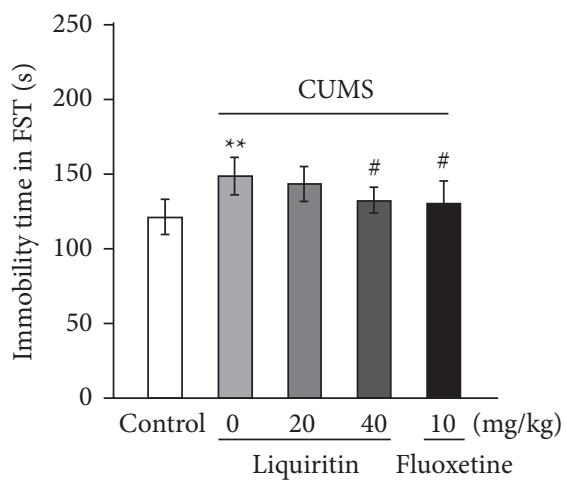

(d)

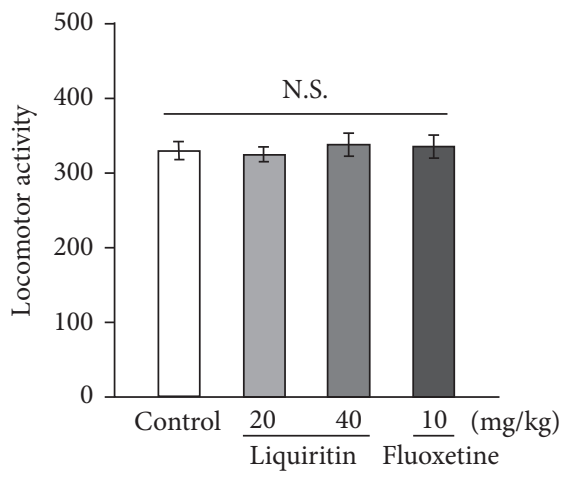

(e)

FIgURE 1: Effects of liquiritin or fluoxetine on desperate behavior in mice. (a) Chemical structure of liquiritin. (b) Schematic diagram of despair experiment. (c) Tail suspension test. (d) Forced swimming test. (e) Locomotor activity. Liquiritin or fluoxetine was administered intragastrically once daily for consecutive 14 days. Data represent means $\pm \mathrm{SD}(n=10)$. For statistical significance, ${ }^{*} P<0.05$ and ${ }^{* *} P<0.01$ versus the control group. N.S.: nonsignificant.

The exploratory behavior was recorded for $5 \mathrm{~min}$, including the open-arm entries and the time spent in each arm. The maze was wiped with $75 \%$ alcohol after each animal was done.

\subsection{Antioxidant Activity and Cytokines Detection.} Hippocampus of mice was homogenized in an ice bath and then centrifuged at $12000 \mathrm{rpm}$ for $15 \mathrm{~min}$. The supernatant was separated and stored at $4^{\circ} \mathrm{C}$. The activities of SOD and GSH-Px and the content of MDA in the hippocampus were determined by ultraviolet spectrophotometry. The levels of IL- $1 \beta$ and IL-18 were measured by ELISA. All operations were carried out according to the kit instructions.
2.10. Western Blot Analysis. The total protein of hippocampal tissue was separated by SDS-PAGE gel electrophoresis and transferred onto polyvinylidene fluoride (PVDF) membrane. The dilution ratios of the antibodies were as follows: NLRP3 antibody and TLR4 antibody $(1: 500)$ and $\beta$-actin antibody, IL- $1 \beta$ antibody, caspase- 1 antibody, pro-caspase- 1 antibody, IL- $1 \beta$ antibody, NEK7 antibody, and ASC antibody $(1: 1000)$. Then, HRP-labeled secondary antibody $(1: 3000)$ was added, incubated for $2 \mathrm{~h}$. The gel imaging system was used to detect and photograph, and the strips were analyzed by ImageJ software (Maryland, USA). $\beta$-Actin was used as an internal reference to calculate relative protein expression. 


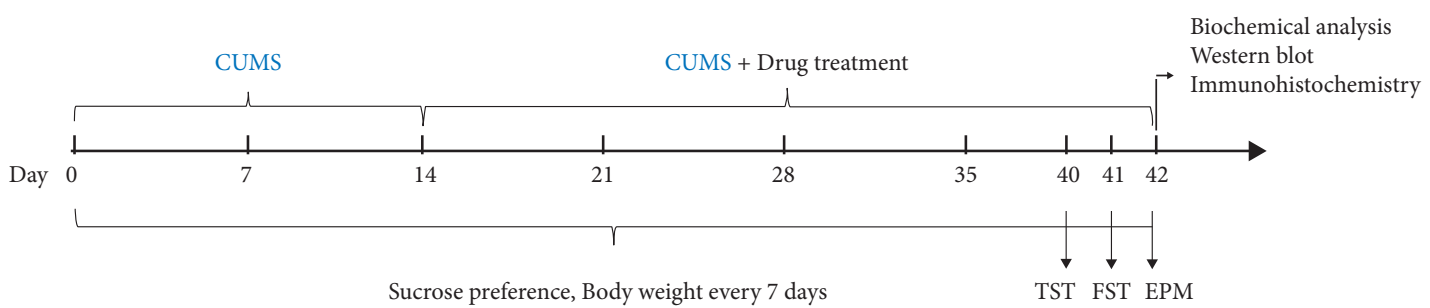

(a)

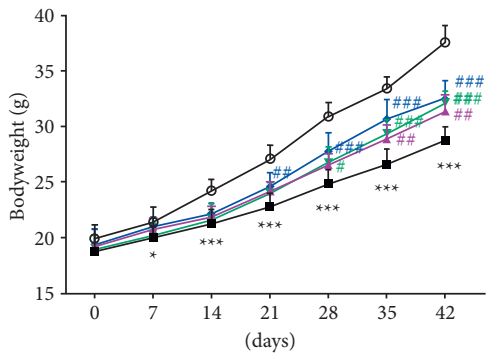

$\rightarrow$ Control

$\rightarrow$ CUMS

$\longrightarrow$ CUMS + Liquiritin $(20 \mathrm{mg} / \mathrm{kg})$

$\rightarrow$ CUMS + Liquiritin $(40 \mathrm{mg} / \mathrm{kg})$

$\multimap$ CUMS + Fluoxetin $(10 \mathrm{mg} / \mathrm{kg})$

(b)

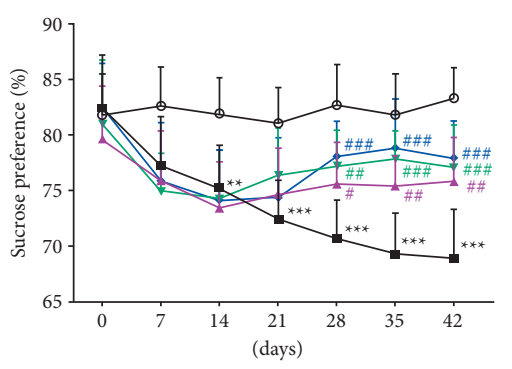

$\rightarrow$ Control

$\rightarrow-$ CUMS

$\multimap$ CUMS + Liquiritin $(20 \mathrm{mg} / \mathrm{kg})$

$\rightarrow$ CUMS + Liquiritin $(40 \mathrm{mg} / \mathrm{kg})$

$\multimap$ CUMS + Fluoxetin $(10 \mathrm{mg} / \mathrm{kg})$

(c)

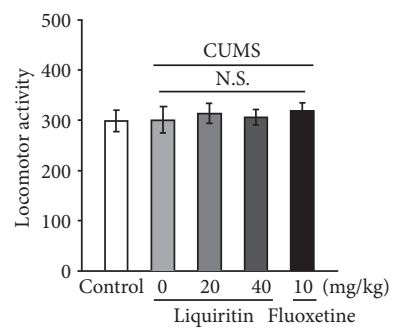

(d)

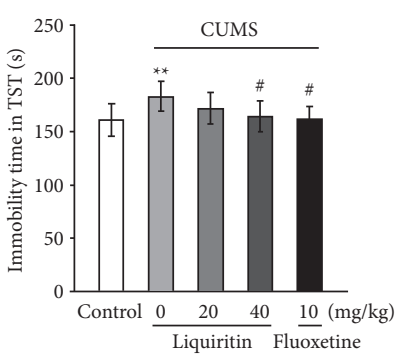

(e)

Figure 2: Continued. 


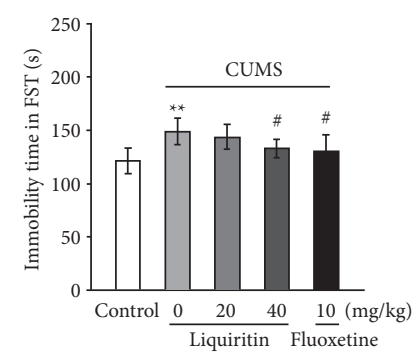

(f)

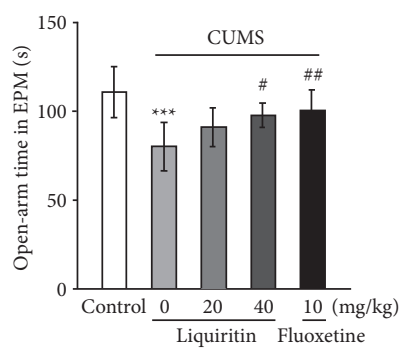

(g)

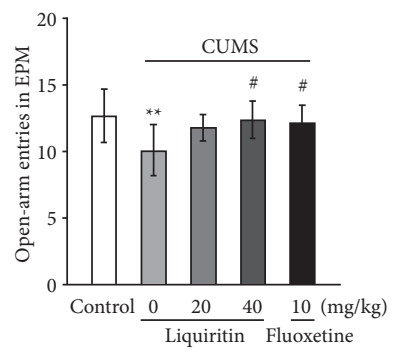

(h)

FIgURE 2: Effects of liquiritin or fluoxetine on depression-like behavior in CUMS mice. (a) CUMS experimental design. (b) Sucrose preference test every 7 days. (c) Bodyweight assessment every 7 days. (d) Locomotor activity in elevated plus-maze. (e) Tail suspension test. (f) Forced swimming test. (g) The total time of mice entered the open-arm in elevated plus-maze. (h) The open-arm entries in elevated plusmaze. Data represent means \pm SD $(n=10)$. For statistical significance, ${ }^{*} P<0.05,{ }^{* *} P<0.01$, and ${ }^{* * *} P<0.001$ versus the control group; ${ }^{\#} P<0.05,{ }^{\#} P<0.01$, and ${ }^{\# \# \#} P<0.001$, versus the CUMS group. N.S.: nonsignificant.

2.11. Immunohistochemistry (IHC). According to the previous method [13], after the mice were killed, hippocampal tissues were quickly removed and soaked overnight in $10 \%$ paraformaldehyde at $4^{\circ} \mathrm{C}$ to make paraffin sections. Rabbit TLR4 antibody $(1: 100)$ and rabbit NLRP3 antibody $(1: 100)$ were used for IHC detection after dewaxing treatment. The sections were washed and incubated with the secondary antibody $(1: 3000)$. Subsequently, an image of the hippocampal CA3 region was obtained using an optical microscope and analyzed by ImageJ software.

\subsection{Cellular Thermal Shift Assay (CETSA). According to} the documentary method [14], $2 \times 10^{6}$ PC12 cells and $8 \mathrm{ml}$ RPMI-1640 were separately added in $\varphi 100 \mathrm{~mm}$ dishes. After incubation for $24 \mathrm{~h}$, liquiritin was added at a final concentration of $200 \mu \mathrm{m}$, and DMSO was used as a control. With a continuous incubation for $2 \mathrm{~h}$, cells were collected. After centrifugation, cell lysates were aliquoted and individually heated to the indicated temperatures (ranging from $25^{\circ} \mathrm{C}$ to $65^{\circ} \mathrm{C}$ ) for $3 \mathrm{~min}$ and then cooled at room temperature for $3 \mathrm{~min}$, repeating it for 3 cycles. Insoluble proteins were separated by centrifugation, and then a Western blot analysis was used to detect the changes in the NLRP3 protein.

2.13. Statistical Analysis. Statistical analysis was performed using GraphPad Prism 8.0 software (California, USA). The experimental data were expressed as means \pm standard deviation (SD). One-way ANOVA analysis was used to compare the sample means between groups. $P<0.05$ indicates that the difference is statistically significant.

\section{Results}

3.1. Liquiritin Alleviated the Desperate State and Depressionlike Behavior in Mice. The desperate behavior test was performed to initially evaluate the antidepressant effect of liquiritin in naive mice. As shown in Figures 1(c) and 1(e), after 14 days of intragastric administration with liquiritin ( $40 \mathrm{mg} / \mathrm{kg}$ ), the immobile time of TST and FST was reduced, respectively, compared with the control group. To rule out the interference of liquiritin on central excitability, a 
locomotion activity test was carried out. The results showed that liquiritin showed no significant effect on locomotion activity.

CUMS model was established to further investigate the antidepression effect of liquiritin. The CUMS model was thought to imitate the main symptoms of depression and has been widely used to explore the pathogenesis of depression [15]. Reduced sucrose consumption is regarded as a key indicator in animals [16]. We found that sucrose consumption and body weight decreased significantly from day 14 in the liquiritin-treated group (Figures 2(b) and 2(c)). On days 40 and 41, TST and FST were undertaken. Liquiritin $(40 \mathrm{mg} / \mathrm{kg})$ reduced immobile time in CUMS mice, respectively (Figures 2(e) and 2(f)). On day 42, we performed an elevated plus-maze, widely used to evaluate anxiety and depression in rodents [17]. We observed a significant increase in the time spent and times of entering the open arms in the liquiritin group (Figures $2(\mathrm{~g})$ and $2(\mathrm{~h})$ ). However, there was no significant difference in locomotive activity between the liquiritin and CUMS groups (Figure 2(d)). In all tests, the positive drug fluoxetine showed significant antidepression effects in mice, consistent with previous reports [18]. All the data above indicated that liquiritin attenuated the depression-like behavior of CUMS mice.

\subsection{Liquiritin Enhanced the Antioxidant Capacity of CUMS} Mice. A series of studies have shown that acute and chronic stress trigger oxidative stress damage in brain regions, which is highly associated with depression [19]. It has been confirmed that the depressive behavior of rats is related to the increased level of lipid peroxidation metabolite MDA, suggesting that the harmful effects of CUMS on the mood and behavior of rats are, at least partly, mediated by oxidation [20]. Here, we found that the MDA content of mice increased significantly in CUMS group. In the liquiritin$(40 \mathrm{mg} / \mathrm{kg})$ treated group, SOD activity was increased, and MDA content was decreased in the hippocampus of CUMS mice (Figures 3(a) and 3(b)). However, there was no significant difference in GSH-Px activity among all groups (Figure 3(c)). The above results indicate that liquiritin attenuated oxidative stress and improved the antioxidant capacity of CUMS mice.

3.3. Liquiritin Decreased the Levels of IL-18 and IL-1 $\beta$ in the Hippocampus of CUMS Mice and Inhibited the Expression of NLRP3 Inflammasome Activation. It has been found recently that IL-1 $\beta$ and IL-18 play an important role in depression. In our study, we found that the levels of IL- $1 \beta$ and IL-18 in the hippocampus of CUMS mice were higher than those of the control group (Figures 3(d) and 3(e)). Liquiritin $(40 \mathrm{mg} / \mathrm{kg})$ reduced the contents of IL-18 and IL- $1 \beta$ in the hippocampus of CUMS mice, respectively. In order to clarify the pathway by which liquiritin inhibits inflammatory response, we detected the protein expression of NLRP3 and Toll-like receptor 4 (TLR4), which was considered to contribute to the production and secretion of the mature IL- $1 \beta$. The results showed that liquiritin $(40 \mathrm{mg} / \mathrm{kg})$ downregulated the expression of NLRP3 in the hippocampus of CUMS mice
(Figures 4(a) and 4(b)). By contrast, it showed no significant effect on TLR4 expression (Figures 4(a) and 4(c)), and the IHC data confirmed this result (Figures 4(d)-4(f)). Additionally, we found that liquiritin $(40 \mathrm{mg} / \mathrm{kg})$ downregulated the expression of NLRP3 inflammasome-associated proteins, including ASC, caspase-1, pro-caspase- 1, IL- $1 \beta$, and NEK7 in the hippocampus of CUMS mice (Figure 4(a)). These results suggest that liquiritin inhibited the release of cytokines IL- 18 and IL- $1 \beta$ by inhibiting the NLRP3 inflammasome-mediated inflammatory activity, rather than TLR4 mediated pathway.

The CETSA was used to evaluate the binding ability of drugs to proteins [21]. After treating PC12 cells with liquiritin or DMSO, cell lysates were heated at temperature ranging from $25^{\circ} \mathrm{C}$ to $65^{\circ} \mathrm{C}$. The western blot was conducted using a specific antibody for NLRP3. The results suggested liquiritin did not alter the thermal stability of the NLRP3 protein compared to that of DMSO, implying that liquiritin might not bind to NLPR3 by direct action (Figures 4(g) and 4(h)).

\section{Discussion}

In this study, we mainly found that chronic administration of liquiritin improved depression-like behavior of CUMS mice and inhibited inflammasome activation and downstream inflammatory cytokines levels in the hippocampus. As the thermal stability of NLRP3 protein was not altered by liquiritin, liquiritin might inhibit NLRP3 inflammasome activation by acting indirectly through a potential target.

The main symptoms of depression include low mood, reduced activity, and aversion to activities [22]. The CUMS model successfully simulated these core symptoms of depression, and we therefore employed it to evaluate the antidepressant effects of liquiritin. A significant reduction in the immobility time of TST and FST was found, as well as an increase in sucrose consumption, suggesting that liquiritin enhanced the happiness and social exploration interest of CUMS mice.

Studies have demonstrated that the pathogenesis of depression is related to the inflammatory process in the central nervous system, with changes in the release of cytokines, increased oxidative stress, and regulation of the glutathione system [8,9]. Moreover, clinical observations have identified that depression is associated with elevated levels of inflammatory cytokines in the blood, while anticentral inflammatory strategies were effective in improving depressive symptoms [23]. Depression exhibited increases in IL- $1 \beta$ and IL-18 levels associated with activation of NLRP3 inflammasome [24]. NLRP3 inflammasome consists of three important components: the NLRP3 protein as a sensor, apoptosis-associated speck-like protein containing CARD (ASC) as an adaptor, and caspase-1 as an effector [25]. In the normal state, ASC is located in the nucleus, while NLRP3 and pro-caspase- 1 are present in the cytoplasm. When cells are stimulated by pathogens, NLRP 3 recruit and bind to ASC to form an activated NLRP3 inflammasome complex, consequently resulting in pro-caspase-1 activation [26]. Caspase- 1 cleaves the precursors of IL- $1 \beta$ and IL-18 to produce the corresponding mature cytokines [27]. NIMA- 


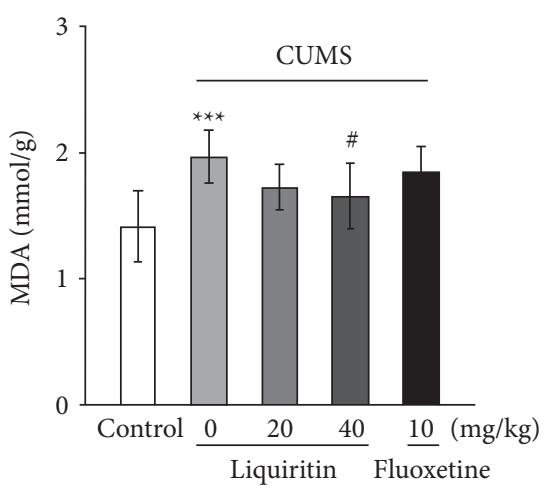

(a)

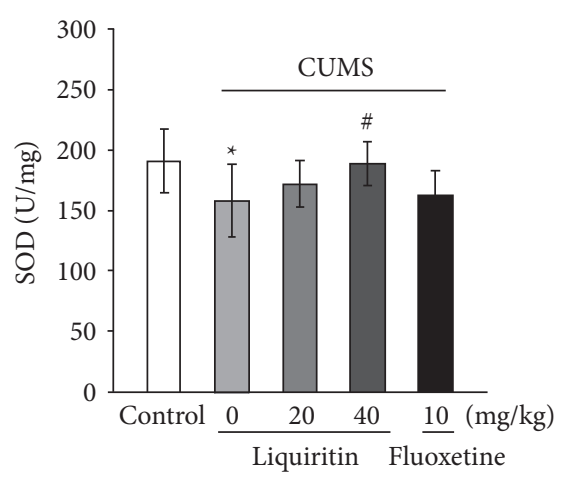

(b)

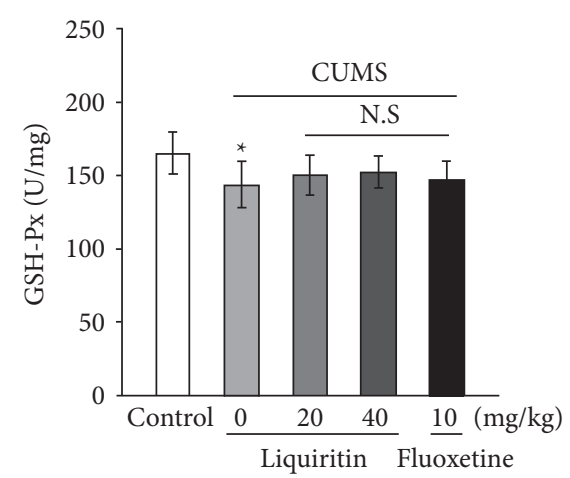

(c)

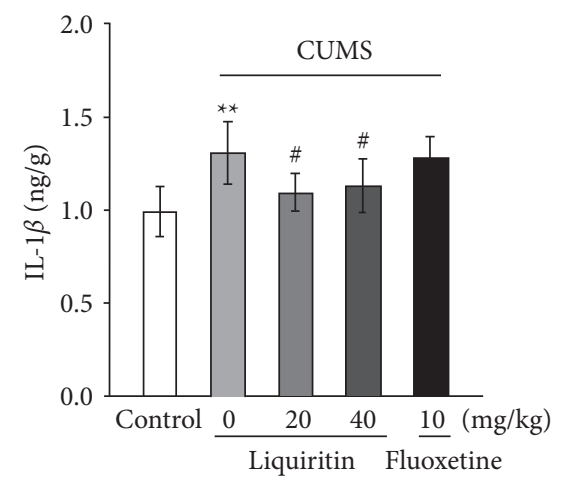

(d)

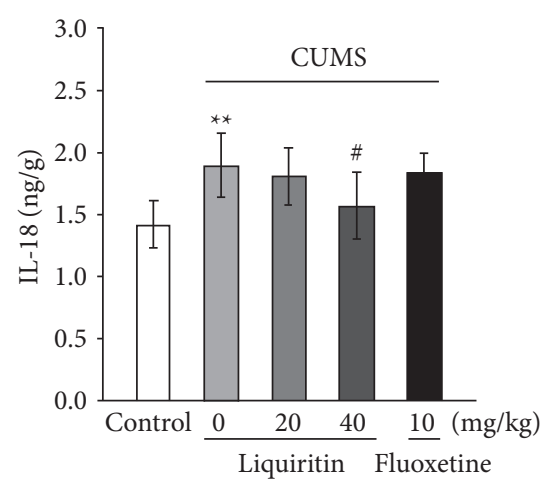

(e)

FIgURE 3: Effects of liquiritin or fluoxetine on antioxidant activity and inflammatory cytokines in hippocampus tissues. (a) MDA levels. (b) SOD activity. (c) GSH-Px content. (d) IL-1 $\beta$ content measured by ELISA. (e) IL-18 content measured by ELISA. Data represent means \pm SD $(n=6)$. For statistical significance, ${ }^{*} P<0.05,{ }^{* *} P<0.01$, and ${ }^{* * *} P<0.001$ versus the control group; ${ }^{\#} P<0.05$ versus the CUMS group. N.S.: nonsignificant.

related kinase 7 (NEK7) is widely expressed in the brain, which is related to the initiation of mitosis, cell cycle progression, and mitotic progression [28]. Studies have found that NEK7 regulated the activation of NLRP3, which played an important role in the inflammatory cascade of macrophages [29]. Here, we show that liquiritin $(40 \mathrm{mg} / \mathrm{kg})$ inhibited the protein expression of NLRP3 inflammasome components in the hippocampal region by western blot and IHC assay, accompanied by significantly lower levels of IL$1 \beta$ and IL-18 as well. The inhibition of NLRP3 inflammasome may be responsible for the antidepressant properties of liquiritin. We also performed CETSA to verify whether there is a direct binding of liquiritin to NLPR3. It was found liquiritin failed to affect the thermal stability of NLRP3 protein, indicating no direct binding of liquiritin to NLRP3 protein. We, therefore, suggest that liquiritin may indirectly inhibit the activation of NLRP3 inflammasome through other potential upstream targets.

In addition to the NLRP3 inflammasome, TLR4 may also be involved in the inhibition of inflammatory cytokines released by liquiritin [30]. When stress is sustained or severe, TLR4 in the cell membrane enhances the expression levels of nuclear factor $-\kappa \mathrm{B}(\mathrm{NF}-\kappa \mathrm{B})$ by both MyD88-dependent and non-MyD88-dependent pathways, which in turn promotes the formation and release of proinflammatory substances such as IL- $1 \beta$ and tumor necrosis factor $\alpha$ (TNF- $\alpha$ ) [31].
Herein, we found that liquiritin had little effect on TLR4 protein expression, suggesting that liquiritin inhibition of IL- $1 \beta$ and IL-18 may be associated with NLRP3 rather than TLR4.

Liquiritin is a natural compound that has been shown to possess anti-inflammatory effect, antioxidant activity, and neuroprotective and antitumor benefits [32-35]. The inhibitory effect of liquiritin on IL- $1 \beta$ was also present in other studies. It has been shown to improve rheumatoid arthritis by reducing VEGF expression in IL- $1 \beta$-induced RA-FLS cells by blocking MAPK signaling pathway [34]. Although liquiritin was not found to downregulate TLR4 expression in the hippocampus of CUMS mice in this study, it has been found to do so in studies of cyclophosphamide-induced hepatotoxicity [33]. In line with most studies, we confirmed the antioxidant effect of liquiritin as evidenced by an increase in hippocampal SOD levels and a decrease in MDA content in CUMS mice. However, to our knowledge, the inhibitory effect of liquiritin on the NLRP3 inflammasome is reported here for the first time. Collectively, our study demonstrates for the first time that liquiritin reduces the release of central cytokines by inhibiting NLRP3 inflammasome. As an intensively studied anti-inflammatory natural compound, liquiritin holds promise for development as an adjuvant antidepressant. 


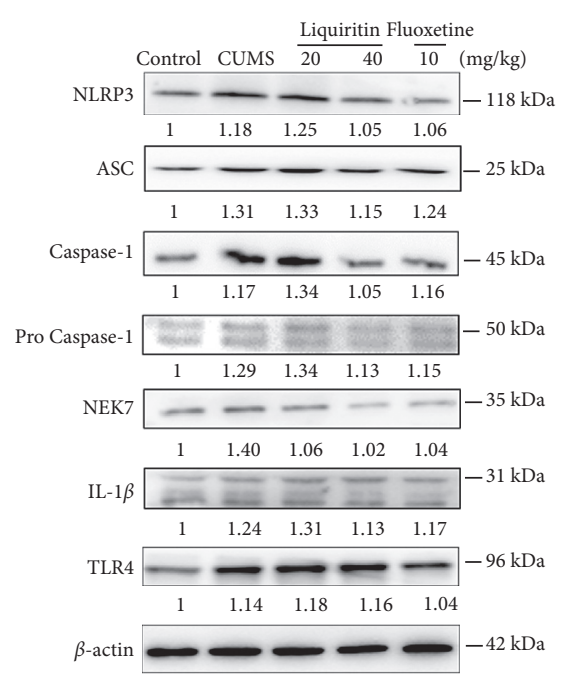

(a)

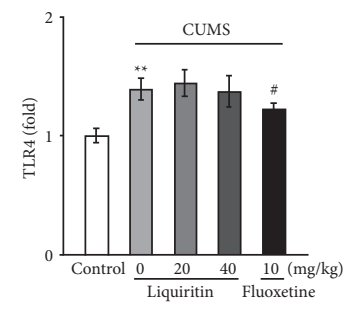

(c)

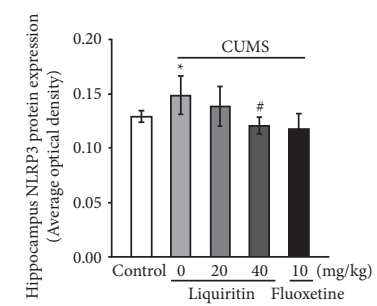

(e)

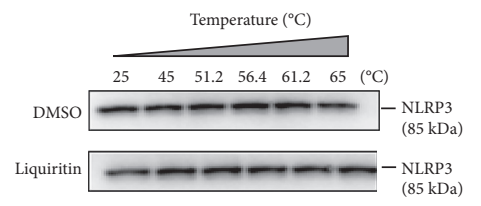

(g)

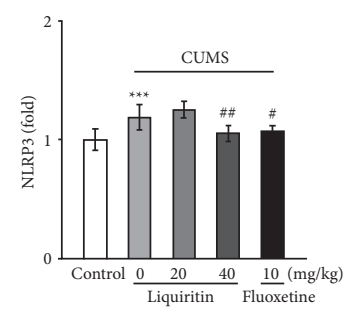

(b)

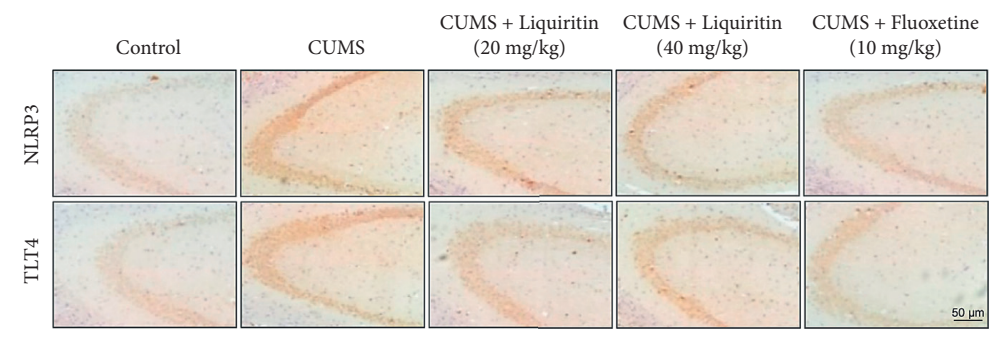

(d)

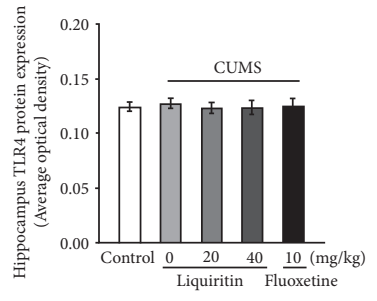

(f)

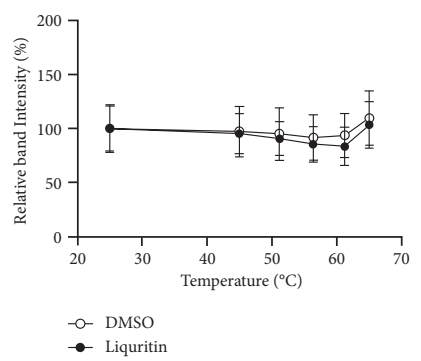

(h)

FIGURE 4: Effects of liquiritin or fluoxetine on the expression of inflammatory proteins and the interactions with proteins. (a) NLRP3 inflammasome-associated protein expression in the hippocampus. $\beta$-Actin was used as an internal reference. (b, c) Quantification of NLRP3 and TLR4 protein expression by Western blot $(n=3)$. (d) Expression of NLRP3 and TLR4 in hippocampal CA3 region measured by IHC. Positive cells are represented as brown spots. (e, f) Quantification of NLRP3 and TLR4 expression by IHC ( $n=3)$. (g, h) CETSA and quantification. Quantification of both Western blot and IHC was performed using ImageJ software. Data represent means \pm SD $(n=3)$. For statistical significance, ${ }^{*} P<0.05,{ }^{* *} P<0.01$, and ${ }^{* * *} P<0.001$ versus control group; ${ }^{\#} P<0.05$ and ${ }^{\# \#} P<0.01$ versus the CUMS group. 


\section{Data Availability}

The datasets used and analyzed during the current study are available from the corresponding author on reasonable request.

\section{Ethical Approval}

All experimental animal protocols were approved by the guidelines of the Changchun University of Chinese Medicine Animal Ethics Committee (No. SYXK (Ji) 2018-0014).

\section{Conflicts of Interest}

The authors declare that there are no conflicts of interest regarding the publication of this article.

\section{Authors' Contributions}

Chang Liu and Dai Yuan contributed equally.

\section{Supplementary Materials}

Figure S1: HPLC analysis of liquiritin. Figure S2: license for the use of laboratory animals. (Supplementary Materials)

\section{References}

[1] M. A. Nettis and C. M. Pariante, "Is there neuroinflammation in depression? understanding the link between the brain and the peripheral immune system in depression," International Review of Neurobiology, vol. 152, pp. 23-40, 2020.

[2] C. Sahin and F. Aricioglu, "Future directions of cytokine hypothesis in depression: "NLRP3 inflamazomu"," Klinik Psikofarmakoloji Bülteni-Bulletin of Clinical Psychopharmacology, vol. 23, no. 3, 2016.

[3] Y. Xu, H. Sheng, Q. Bao, Y. Wang, J. Lu, and X. Ni, "NLRP3 inflammasome activation mediates estrogen deficiency-induced depression- and anxiety-like behavior and hippocampal inflammation in mice," Brain, Behavior, and Immunity, vol. 56, pp. 175-186, 2016.

[4] J.-Y. Yu, J. Ha, K.-M. Kim, Y.-S. Jung, J.-C. Jung, and S. Oh, "Anti-Inflammatory activities of licorice extract and its active compounds, glycyrrhizic acid, liquiritin and liquiritigenin, in BV2 cells and mice liver," Molecules, vol. 20, no. 7, pp. 13041-13054, 2015.

[5] S. H. He, H.-G. Liu, Y.-F. Zhou, and Q.-F. Yue, "Liquiritin (LT) exhibits suppressive effects against the growth of human cervical cancer cells through activating caspase- 3 in vitro and xenograft mice in vivo," Biomedicine \& Pharmacotherapy, vol. 92, pp. 215-228, 2017.

[6] H. Shi, Y. Wu, Y. Wang et al., "Liquiritigenin potentiates the inhibitory effects of cisplatin on invasion and metastasis via downregulation MMP-2/9 and PI3 K/AKT signaling pathway in B16F10 melanoma cells and mice model," Nutrition and Cancer, vol. 67, no. 5, pp. 761-770, 2015.

[7] Z. A. Chen, J. L Wang, R. T Liu et al., "Liquiritin potentiate neurite outgrowth induced by nerve growth factor in PC12 cells," Cytotechnology, vol. 60, no. 1-3, pp. 125-132, 2009.

[8] Z. Kawakami, Y. Ikarashi, and Y. Kase, "Isoliquiritigenin is a novel NMDA receptor antagonist in kampo medicine yokukansan," Cellular and Molecular Neurobiology, vol. 31, no. 8, pp. 1203-1212, 2011.
[9] W. Wang, X. Hu, Z. Zhao et al., “Antidepressant-like effects of liquiritin and isoliquiritin from glycyrrhiza uralensis in the forced swimming test and tail suspension test in mice," Progress in Neuro-Psychopharmacology and Biological Psychiatry, vol. 32, no. 5, pp. 1179-1184, 2008.

[10] Z. Zhao, W. Wang, H. Guo, and D. Zhou, "Antidepressantlike effect of liquiritin from glycyrrhiza uralensis in chronic variable stress induced depression model rats," Behavioural Brain Research, vol. 194, no. 1, pp. 108-113, 2008.

[11] T. Zou, K. Sugimoto, J. Zhang et al., "Geniposide alleviates oxidative stress of mice with depression-like behaviors by upregulating six3os1," Frontiers in Cell and Developmental Biology, vol. 8, Article ID 553728, 2020.

[12] L. X. Chen, Z. Qi, Z. J. Shao et al., "Study on antidepressant activity of pseudo-ginsenoside HQ on depression-like behavior in mice," Molecules (Basel, Switzerland), vol. 24, no. 5, 2019.

[13] H. A. Bedel, C. Kencebay Manas, G. Özbey, and C. Usta, "The antidepressant-like activity of ellagic acid and its effect on hippocampal brain derived neurotrophic factor levels in mouse depression models," Natural Product Research, vol. 32, no. 24, pp. 2932-2935, 2018.

[14] Y. Li, Y. Zhang, L. Wang et al., “Autophagy impairment mediated by S-nitrosation of ATG4B leads to neurotoxicity in response to hyperglycemia," Autophagy, vol. 13, no. 7, pp. 1145-1160, 2017.

[15] K. Xu, Z. Liu, L. Wang, G. Wu, and T. Liu, "Influence of hippocampal low-frequency stimulation on GABAA $\mathrm{R} \alpha 1$, ICER and BNDF expression level in brain tissues of amygdalakindled drug-resistant temporal lobe epileptic rats," Brain Research, vol. 1698, pp. 195-203, 2018.

[16] P. Willner, "Reliability of the chronic mild stress model of depression: a user survey," Neurobiology of Stress, vol. 6, pp. 68-77, 2017.

[17] P. Willner, "The chronic mild stress (CMS) model of depression: history, evaluation and usage," Neurobiology of Stress, vol. 6, pp. 78-93, 2017.

[18] R. Andreatini and L. F. S. Bacellar, "Animal models: trait or state measure? the test-retest reliability of the elevated plusmaze and behavioral despair," Progress in Neuro-Psychopharmacology and Biological Psychiatry, vol. 24, no. 4, pp. 549-560, 2000.

[19] S. Marwari and G. S. Dawe, "(R)-fluoxetine enhances cognitive flexibility and hippocampal cell proliferation in mice," Journal of Psychopharmacology, vol. 32, no. 4, pp. 441-457, 2018.

[20] L. A. D. Silva, L. Tortelli, J. Motta et al., "Effects of aquatic exercise on mental health, functional autonomy and oxidative stress in depressed elderly individuals: a randomized clinical trial," Clinics (Sao Paulo, Brazil), vol. 74, p. e322, 2019.

[21] C. Du, C. Xi, C. Wu, J. Sha, J. Zhang, and C. Li, "Ginkgo biloba extract protects early brain injury after subarachnoid hemorrhage via inhibiting thioredoxin interacting protein/NLRP3 signaling pathway," Iranian journal of basic medical sciences, vol. 23, no. 10, pp. 1340-1345, 2020.

[22] R. Pine, T. Fleming, S. McCallum, and K. Sutcliffe, "The effects of casual videogames on anxiety, depression, stress, and low mood: a systematic review," Games for Health Journal, vol. 9, no. 4, pp. 255-264, 2020.

[23] S. Liu, X. Deng, P. Zhang et al., "Blood flow patterns regulate PCSK9 secretion via MyD88-mediated pro-inflammatory cytokines," Cardiovascular Research, vol. 116, no. 10, pp. 1721-1732, 2020. 
[24] H. S. Foyet, S. Tchinda Deffo, P. Koagne Yewo et al., "Ficus sycomorus extract reversed behavioral impairment and brain oxidative stress induced by unpredictable chronic mild stress in rats," BMC Complementary and Alternative Medicine, vol. 17, no. 1, p. 502, 2017.

[25] J.-Y. Li, Y.-Y. Wang, T. Shao et al., "The zebrafish NLRP3 inflammasome has functional roles in ASC-dependent interleukin-1 $\beta$ maturation and gasdermin E-mediated pyroptosis," Journal of Biological Chemistry, vol. 295, no. 4, pp. 1120-1141, 2020.

[26] J. Rong, J. Xu, Q. Liu et al., "Anti-inflammatory effect of upregulated microRNA-221-3p on coronary heart disease via suppressing NLRP3/ASC/pro-caspase-1 inflammasome pathway activation," Cell Cycle, vol. 19, no. 12, pp. 1478-1491, 2020.

[27] K. Sarantis, K. Antoniou, N. Matsokis, and F. Angelatou, "Exposure to novel environment is characterized by an interaction of D1/NMDA receptors underlined by phosphorylation of the NMDA and AMPA receptor subunits and activation of ERK1/2 signaling, leading to epigenetic changes and gene expression in rat hippocampus," Neurochemistry International, vol. 60, no. 1, pp. 55-67, 2012.

[28] H. Cai, P. Wang, B. Zhang, and X. Dong, "Expression of the NEK7/NLRP3 inflammasome pathway in patients with diabetic lower extremity arterial disease," BMJ Open Diabetes Research \& Care, vol. 8, no. 2, 2020.

[29] I. Boal-Carvalho, B. Mazel-Sanchez, F. Silva et al., "Influenza A viruses limit NLRP3-NEK7-complex formation and pyroptosis in human macrophages," EMBO Reports, vol. 21, no. 12, Article ID e50421, 2020.

[30] F. N. Kaufmann, A. P. Costa, G. Ghisleni et al., "NLRP3 inflammasome-driven pathways in depression: clinical and preclinical findings," Brain, Behavior, and Immunity, vol. 64, pp. 367-383, 2017.

[31] X. He, Z. Wei, J. Wang et al., "Alpinetin attenuates inflammatory responses by suppressing TLR4 and NLRP3 signaling pathways in DSS-induced acute colitis," Scientific Reports, vol. 6, no. 1, Article ID 28370, 2016.

[32] X. Yang, X. Dang, X. Zhang, and S. Zhao, "Liquiritin reduces lipopolysaccharide-aroused $\mathrm{HaCaT}$ cell inflammation damage via regulation of microRNA-31/MyD88," International Immunopharmacology, vol. 101, no. Pt B, Article ID 108283, 2021.

[33] M. Chen, C. Zhang, J. Zhang et al., "The involvement of DAMPs-mediated inflammation in cyclophosphamide-induced liver injury and the protection of liquiritigenin and liquiritin," European Journal of Pharmacology, vol. 856, Article ID 172421, 2019.

[34] K. F. Zhai, H. Duan, C.-Y. Cui et al., "Liquiritin from glycyrrhiza uralensis attenuating rheumatoid arthritis via reducing inflammation, suppressing angiogenesis, and inhibiting MAPK signaling pathway," Journal of Agricultural and Food Chemistry, vol. 67, no. 10, pp. 2856-2864, 2019.

[35] X. Q. Li, L. M. Cai, J. Liu et al., "Liquiritin suppresses UV binduced skin injury through prevention of inflammation, oxidative stress and apoptosis through the TLR4/MyD88/ NFkappaB and MAPK/caspase signaling pathways," International Journal of Molecular Medicine, vol. 42, no. 3, pp. 1445-1459, 2018. 